\title{
Avascular necrosis in proximal humeral fractures in patients treated with operative fixation: a meta-analysis
}

\author{
Jiaming $\mathrm{Xu}^{\dagger}$, Changqing Zhang ${ }^{\dagger}$ and Tao Wang ${ }^{*}$
}

\begin{abstract}
Background: Proximal humeral fractures are common lesions of the elderly, but there are no established treatment guidelines. A surgical treatment for comminuted and displaced fractures of the proximal humerus was developed and is still evolving. The aim of this study was to perform a quantitative review to evaluate the risk of avascular necrosis (AVN) in patients with proximal humeral fractures who were treated by operative fixation compared with conservative treatment.

Methods: We searched the PubMed, MEDLINE, Springer, Elsevier Science Direct, Cochrane Library, Google Scholar, China National Knowledge Infrastructure (in Chinese), and Wanfang database (in Chinese) up to December 2013 to identify studies related to operative fixation and AVN in patients with proximal humeral fractures.

Results: Seven studies with a total of 291 patients (142 operative fixation cases and 149 conservative treatment cases) with proximal humeral fractures were considered in the meta-analysis. The overall meta-analysis showed no significant difference in the incidence of AVN between the two groups [odds ratio (OR) 1.42, 95\% confidence interval (Cl) $0.33-6.11, p=0.64]$. The subgroup meta-analysis by study design (retrospective/prospective), sample size ( $\leq 40 />40$ ), and ethnicity (European/Asian) demonstrated similar results. However, the subgroup analysis by specific operative approach (plate fixation/tension band wiring fixation/others) indicated that plate fixation was associated with a higher rate of AVN than conservative treatment (OR 0.20,95\% Cl $0.05-0.76, p=0.019)$.
\end{abstract}

Conclusions: Plate fixation was associated with a higher risk of AVN development than conservative treatment in patients with proximal humeral fractures.

Keywords: Avascular necrosis, Operative fixation, Conservative treatment, Proximal humeral fracture, Meta-analysis

\section{Background}

Although proximal humeral fractures are common lesions of the elderly, accounting for $5 \%$ of all fractures and $45 \%$ of all humeral fractures [1-3], there is no consensus regarding their treatment. The majority of patients suffering this injury have nondisplaced or minimally displaced twoor three-part fractures. They are treated conservatively with a modified Velpeau bandage or in a sling, both of which require immobilization for more than 2 weeks $[4,5]$. This conservative treatment of severe displacement of proximal humeral fracture fragments, however, often

\footnotetext{
* Correspondence: taowang2@outlook.com

${ }^{\dagger}$ Equal contributors

Department of Orthopedics, Shanghai Jiaotong University Affiliated Sixth People's Hospital, 600 Yishan Road, Shanghai 200233, China
}

yields poor functional results [6]. Therefore, operative treatment of comminuted and displaced fractures of the proximal humerus was developed and has been evolving in recent years [7].

Currently, many surgical solutions such as operative fixations (retrograde percutaneous pin fixation [5], transcutaneous fixation with Kirschner wires [8], and tension band osteosynthesis [9]) and hemiarthroplasty [10] are applied to treat these lesions, ranging from percutaneous pinning to shoulder arthroplasty [1]. These surgical solutions have added to the surgeon's armamentarium of methods to treat proximal humeral fractures and have been suitable for patients with various conditions. Surgical solutions, however, do little to limit the activities of patients compared with the conservative approach. Also, 
open operative techniques pose a higher risk of the patient developing avascular necrosis (AVN) of the humeral head [6].

Whether the incidence of AVN is less after operative fixation versus conservative treatment for proximal humeral fractures is controversial. In a multicenter analysis reported by Schai et al., open reduction and internal fixation was associated with significantly less AVN than conservative treatment of humeral head fractures [11]. In contrast, Fjalestad and coworkers reported that surgical treatment of displaced proximal humeral fractures was associated with obviously more AVN development than conservative treatment [5].

There was thus a need to gather the current best evidence of AVN incidence in patients with proximal humeral fractures in regard to operative fixation versus conservative treatment. Therefore, we conducted a systematic review of published research and applied a metaanalysis to integrate the results quantitatively.

\section{Methods}

Source of material

We used established search strategies and retrieved literature in a systematic way from various databases, including PubMed, MEDLINE, Springer, Elsevier Science Direct, Cochrane Library, Google Scholar, CNKI (China National Knowledge Infrastructure, in Chinese), and Wanfang (in Chinese) up to December 2013. The key words 'avascular necrosis', 'operative fixation', and 'proximal humeral fractures' were used for the searches. References from retrieved papers were checked for additional studies. We collected data only from fully published papers-not from meeting or conference abstracts. The publication date was not restricted in our research.

\section{Inclusion and exclusion criteria}

Studies were included in the meta-analysis if they met the following criteria. The study had to contain (1) the occurrence rates of AVN in patients with proximal humeral fractures (prospective, retrospective, and crosssectional studies), (2) operative fixation versus conservative treatment data, and (3) the effect size reported as odds ratios (ORs) and 95\% confidence intervals (CIs) or it could be calculated. The sample size and age range were not limited. Studies were excluded if the study (1) described only conservative treatment data in a review or report, (2) was a reduplicated study, or (3) did not compare operative fixation with conservative treatment.

\section{Data extraction and quality evaluation}

Articles were reviewed and filtered out independently by two investigators according to our criteria. The data were then extracted independently in duplicate using a standardized form to assess eligibility for inclusion. Data items included study details (e.g., the first author's name, research year(s) of the study, year of the study's publication, location of participants, design of the study, follow-up time) and characteristics of the participants (e.g., age, sex, sample size). Discrepancies were resolved by consensus.

Evaluation of quality mainly included the sample size and recruitment of the studies. The papers were first selected by reading the document titles and abstracts. Then, we read the full text of each paper to determine whether the study conformed to our inclusion criteria. Two investigators independently completed this task. Assuming that differences would occur, agreements were to be reached by discussion.

\section{Meta-analysis methods}

The meta-analysis was performed in fixed or random effect models as appropriate. The effect sizes of the ORs with 95\% CIs were pooled to assess the AVN incidence associated with operative fixation in proximal humeral fractures compared with conservative treatment. Heterogeneity among studies was evaluated by Cochran's $Q-$ statistic [12] and $I^{2}$ parameter testing [13]. Also, $p<0.05$ or $I^{2}>50 \%$ was considered to indicate a heterogeneous nature. When substantial heterogeneity was detected, we calculated the summary ORs and their 95\% CIs with the DerSimonian and Laird method in the random effect model [14]. If heterogeneity was not detected, the pooled estimate was presented based on the Mantel-Haenszel method in the fixed effect model [15]. To test the reliability of the results, we undertook a sensitivity analysis by repeating the meta-analysis after removing one study each time.

We further conducted subgroup analysis according to the study design (retrospective/prospective), sample size $(\leq 40 />40)$, ethnicity (European/Asian), and specific operative approach (plate fixation/tension band wiring fixation/ others) to investigate the impact of study characteristics on our outcomes. Publication bias was also assessed by Egger's regression asymmetry test [16].

Analyses were performed using the software Review Manager 5.1 (Cochrane Collaboration: http://ims.cochrane. org/revman) and the STATA software package version 11.0 (Stata, College Station, TX, USA). All $p$ values were twosided. A value of $p<0.05$ was considered to indicate statistical significance.

\section{Results}

\section{Characteristics of eligible studies}

The details of the literature search are presented in a flow diagram (Figure 1). We identified 1,120 papers potentially relevant to the search terms (PubMed, 245; MEDLINE, 147; Springer, 276; Elsevier Science Direct, 132; Cochrane Library, 19; Google Scholar, 165; Wanfang, 57; CNKI, 79). There were 117 studies after removing duplicates. During the screening phase based on titles and abstracts, 123 


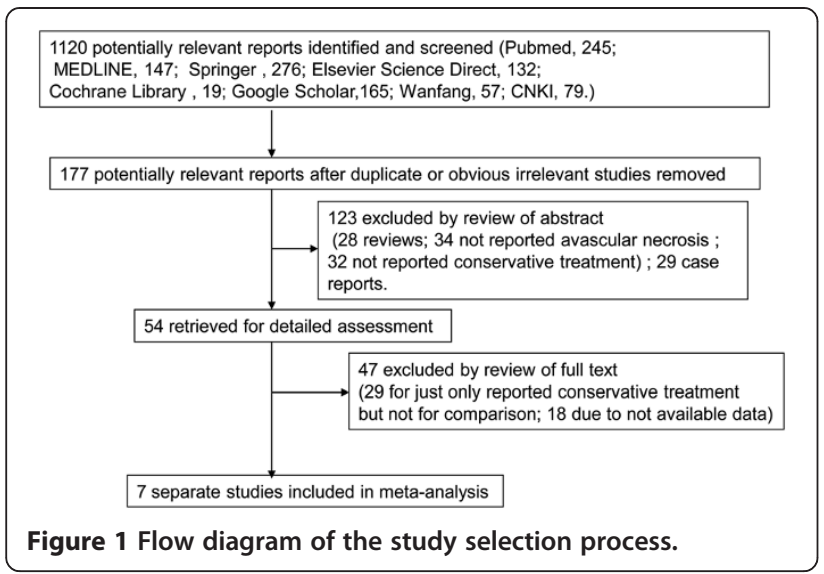

articles were excluded (28 were review articles; 34 did not report AVN; 32 did not report conservative treatment; 29 were case reports). Among the remaining 54 studies for full publication review, only 7 met the inclusion criteria after full publication review.

The characteristics of the seven included studies $[5,9,11,17-20]$ are presented in Table 1 . The studies were published between 1988 and 2012. A total of 291 patients (142 operative fixation cases and 149 conservatively treated cases) with proximal humeral fractures were considered in this meta-analysis. The studies' sample sizes ranged between 12 and 93, and patients' ages ranged between 65.2 and 75.0 years. Various operative approaches were conducted. Plate fixation was performed in two of the seven studies $[11,20]$, tension band wiring fixation in two studies $[9,18]$, and Steinmann pin fixation in one study [17]. Also, AO angular blade plate, screw fixation and cerclage, antegrade screw nails, and retrograde pin fixation were used in one study each [5]. Data for single operative approaches were not provided. In the remaining article [19], no details were given for the open reduction with internal fixation approach.

\section{Overall analysis}

In an overall analysis of the seven selected studies, the heterogeneity test showed that there were heterogeneities
$\left(Q^{2}=16.68, I^{2}=64 \%, p=0.01\right)$ among studies. Thus, the random effect model was applied. The results showed that there was a relatively higher risk of AVN in patients with proximal humeral fractures who underwent operative fixation than in those treated conservatively (Figure 2) (total $\mathrm{OR}=1.42,95 \%$ CI $0.33-6.11, p>0.05$ ), but the difference was not statistically significant. The result of Egger's linear regression test (Table 2) indicated that there was no publication bias in this study $(t=1.52, p>0.05)$.

\section{Subgroup analysis}

The outcomes of the subgroup analysis stratified by study design, sample size, ethnicity, and operative approach are shown in Table 2. The results indicated that a nonsignificant increase of AVN in patients with proximal humeral fractures was consistent in the subgroup analyses when stratified by sample size. In the study design analysis, patients in retrospective studies showed that operative fixation had a nonsignificantly decreased risk of AVN (OR $0.73,95 \% \mathrm{CI} 0.15-3.55, p>0.05)$. In the stratified analysis by ethnicity, Asian patients (OR 0.74, 95\% CI 0.04-12.82) showed less risk of developing AVN than European patients (OR 1.60, 95\% CI 0.30-8.51) when treated with operative fixation. The result of the subgroup analysis by operative approach demonstrated that plate fixation had a significantly increased risk of AVN compared with conservative treatment (OR $0.20,95 \%$ CI $0.05-0.76, p=0.019$ ), whereas tension band wiring fixation showed a risk similar to that with conservative treatment (OR 1.77, 95\% CI $0.41-7.70, p>0.05)$.

\section{Sensitivity analysis and publication bias}

We performed the sensitivity analysis by removing one study each time and rerunning the model to determine the effect on each overall estimate. By omitting the Fjalestad et al. study [5], for instance, the pooled OR was changed to 0.64 (95\% CI $0.29-1.44, p>0.05)$, and the heterogeneity test showed that there were heterogeneities $\left(I^{2}=38 \%, p=0.15\right)$. Also, the estimates changed little, which implied that our results were statistically reliable.

Table 1 Characteristics of studies included in the meta-analysis

\begin{tabular}{|c|c|c|c|c|c|c|}
\hline Study & Country & Ethnicity & Sample size & Study design & Operative approaches & $\begin{array}{c}\text { Average } \\
\text { age, years }\end{array}$ \\
\hline Fan Y, 2012 [20] & China & Asian & 35 & Retrospective & Open reduction and locking plate internal fixation & 75.0 \\
\hline Fjalestad T, 2005 [5] & Norway & European & 51 & $\begin{array}{l}\text { Retrospective and } \\
\text { prospective }\end{array}$ & $\begin{array}{l}\text { AO angular blade plate, screw fixation and cerclage, } \\
\text { antegrade screw nails, or retrograde pin fixation }\end{array}$ & 75.0 \\
\hline Ilchmann T, 1998 [9] & Sweden & European & 24 & Retrospective & Tension band wiring fixation & 65.2 \\
\hline Kristiansen B, 1988 [17] & Denmark & European & 24 & Prospective & Steinmann pin & 69.0 \\
\hline Schai P, 1995 [11] & Switzerland & European & 93 & Retrospective & Minimal internal fixation or plate fixation & 67.5 \\
\hline Zyto K, 1995 [19] & Sweden & European & 35 & Retrospective & Open reduction and internal fixation, not in detail & 71.0 \\
\hline Zyto K, 1997 [18] & Sweden & European & 29 & Prospective & Tension band wiring fixation & 74.0 \\
\hline
\end{tabular}




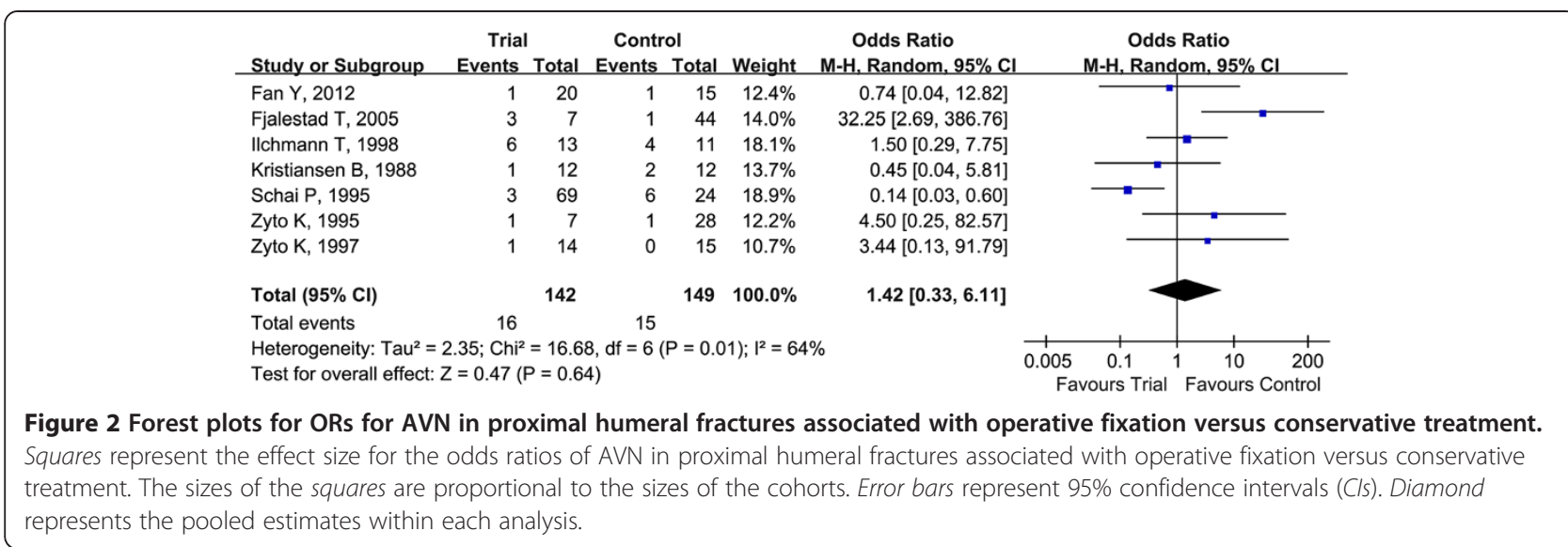

Egger's linear regression test was performed to assess the publication bias of the articles. In this study, the total $p$ value for AVN was 0.19 , which indicated no statistical significance for publication bias.

\section{Discussion}

Previous studies $[5,9,11,18,21]$ have reported the risk of AVN after operative fixation versus conservative treatment in patients with proximal humeral fractures. These studies showed mixed results because of their small sample sizes or low statistical power. In the present meta-analysis, we combined and reanalyzed seven studies that contained 291 patients (142 operative fixation cases and 149 conservatively treated cases) achieve an integrative knowledge of AVN risk with operative fixation.

Findings from the meta-analysis showed that in patients with proximal humeral fractures, operative fixation was associated with a nonsignificantly higher risk of AVN development than conservative treatment (OR 1.42, 95\% CI 0.33-6.11, $p>0.05)$. This finding was consistent with results in a study by Ilchmann and coworkers, who found that the risk of developing AVN was at an OR $(95 \% \mathrm{CI})$ of $1.50(0.29-7.75)$ [9]. Also, the effect sizes of our metaanalysis were similar to those of Kollig and his colleagues' results (OR 1.05, 95\% CI 0.14-8.02) [21]. According to the subgroup analysis, Asian patients (OR 0.74, 95\% CI 0.0412.82 ) and patients in retrospective studies (OR 0.73, 95\% CI $0.15-3.55, p>0.05$ ) showed that operative fixation was associated with a nonsignificantly decreased risk of AVN, which was inconsistent with the overall estimates. Hence, study design and ethnicity might be factors that cause heterogeneity.

Although the overall analysis demonstrated a nonsignificant difference in the incidence of AVN after operative

Table 2 Pooled odds ratio for operative fixation versus conservative treatment in the meta-analysis

\begin{tabular}{|c|c|c|c|c|c|c|c|c|c|c|c|}
\hline \multirow[t]{2}{*}{ Subgroups } & \multicolumn{2}{|c|}{ Sample size } & \multirow[t]{2}{*}{$\begin{array}{l}\text { Number of } \\
\text { studies }\end{array}$} & \multicolumn{3}{|c|}{ Random model } & \multicolumn{3}{|c|}{ Test of heterogeneity } & \multicolumn{2}{|c|}{$\begin{array}{l}\text { Egger's test for } \\
\text { publication bias }\end{array}$} \\
\hline & Case & Control & & OR $(95 \% \mathrm{Cl})$ & $Z$ & $p$ value & $Q$ & $p$ value & $I^{2}(\%)$ & $t$ & $p$ value \\
\hline Overall effects & 142 & 149 & 7 & $1.42(0.33-6.11)$ & 0.47 & 0.64 & 16.68 & 0.01 & 64 & 1.52 & 0.19 \\
\hline \multicolumn{12}{|l|}{ Study design } \\
\hline Retrospective & 109 & 78 & 4 & $0.73(0.15-3.55)$ & 1.21 & 0.23 & 6.89 & 0.08 & 56 & 0.99 & 0.43 \\
\hline Prospective & 26 & 27 & 2 & $1.03(0.17-6.40)$ & 0.03 & 0.98 & 0.92 & 0.34 & 0.0 & -0.03 & 0.98 \\
\hline \multicolumn{12}{|l|}{ Sample size } \\
\hline Sample size $\leq 40$ & 66 & 81 & 5 & $1.36(0.48-3.86)$ & 0.60 & 0.55 & 1.86 & 0.76 & 0.0 & 0.30 & 0.79 \\
\hline Sample size $>40$ & 102 & 87 & 3 & $1.52(0.10-24.11)$ & 0.29 & 0.77 & 14.35 & 0.001 & 86.1 & - & - \\
\hline \multicolumn{12}{|l|}{ Ethnicity } \\
\hline European & 122 & 134 & 6 & $1.60(0.30-8.51)$ & 0.55 & 0.58 & 16.64 & 0.005 & 70 & 1.62 & 0.18 \\
\hline Asian & 20 & 15 & 1 & $0.74(0.04-12.82)$ & 0.21 & 0.83 & - & - & - & - & - \\
\hline \multicolumn{12}{|l|}{ Operative approaches } \\
\hline Plate fixation & 89 & 39 & 2 & $0.20(0.05-0.76)$ & 2.35 & 0.019 & 1.01 & 0.315 & 0.8 & - & - \\
\hline Tension band wiring fixation & 27 & 26 & 2 & $1.77(0.41-7.70)$ & 0.76 & 0.445 & 0.20 & 0.657 & 0.0 & - & - \\
\hline Others & 26 & 84 & 3 & $4.01(0.31-45.26)$ & 1.07 & 0.285 & 5.68 & 0.058 & 64.8 & 0.03 & 0.983 \\
\hline
\end{tabular}


fixation versus conservative treatment, the subgroup analysis by operative approach showed a markedly increased risk of AVN after plate fixation compared with conservative treatment. Tension band wiring fixation and conservative treatment had similar incidences of AVN. Multiple materials have been developed for open reduction and internal fixation including cerclage wires, rotary self-locking intramedullary nails, intramedullary interlocking nails, and six-hole dynamic compression plates [22-24]. Among them, implant of a plate requires a long incision and excision of the perichondrium, causing exposure of soft tissue and even damage to the blood supply, which may contribute to the incidence of AVN.

Treatment of displaced proximal humeral fractures remains a challenge for the orthopedic surgeon [25], and there is no generally accepted strategy for treating them. Procedures using closed reduction are of course less invasive, but it is more difficult to achieve good reconstruction. On the other side, open techniques are more likely to obtain anatomical reduction and stronger fixation, but they present a higher rate of complications related to complete or partial necrosis of the humeral head, regardless of the implant used [1].

The treatment and prognosis of proximal humeral fractures have improved increasingly with the development of medical technology. The study of Poeze et al. indicated that radiographic evaluation in patients with minimally displaced proximal humeral fractures is helpful for predicting functional outcome during conservative treatment [26]. Humeral head replacement was said to be indicated for complex proximal humeral fractures with an avascular head fragment and for those with an unreconstructable fracture [27]. In addition, arthroscopy has become increasingly established in the treatment of proximal humeral fractures [28]. Therefore, the application of operative fixation in proximal humeral fractures should be further studied.

The present study was an updated meta-analysis on the risk of AVN after operative fixation of a proximal humeral fracture. There are some limitations of this study. First, only seven published studies were included in the meta-analysis. Second, significant heterogeneities were detected in the meta-analysis for clinical neurological outcome, which might be distorting the outcomes as heterogeneity is one of the major concerns in meta-analyses regarding validity [29]. Also, different ethnicities may contribute to the heterogeneity, and the patients from each country are not uniform. Therefore, the results should be interpreted with caution. Finally, the recruited studies were not all randomized controlled trials, and some of the sample sizes of the retrieved studies were small [12]. Thus, more high-quality studies are needed to obtain a more integrated, clear outcome.

\section{Conclusions}

The findings of the present meta-analysis suggested that plate fixation in patients with proximal humeral fractures had a significantly higher risk of AVN development than conservative treatment, whereas tension band wiring fixation and conservative treatment had similar risks. Thus, further investigation with more high-quality studies should be carried out to obtain a more precise outcome.

\section{Competing interests}

The authors declare that they have no competing interests.

\section{Authors' contributions}

JX and CZ participated in the design of this study and performed the statistical analysis. JX carried out the study together with $C Z$, collected important background information, and drafted the manuscript. TW conceived of this study, participated in its design, and helped draft the manuscript. All authors read and approved the final manuscript.

\section{Acknowledgements}

This work was supported by the Science and Technology Commission of Shanghai Municipality, China (10DZ1950300). We thank all respondents of the study and all of the people who helped us with the study.

Received: 10 October 2013 Accepted: 11 April 2014

Published: 27 April 2014

\section{References}

1. Imarisio D, Trecci A, Sabatini L, Scagnelli R: Treatment for proximal humeral fractures with percutaneous plating: our first results. Musculoskelet Surg 2013, 1-7.

2. Edwards SL, Wilson NA, Zhang L-q, Flores S, Merk BR: Two-part surgical neck fractures of the proximal part of the humerus. A biomechanical evaluation of two fixation techniques. J Bone Joint Surg 2006 , 88:2258-2264

3. Foruria AM, Carrascal MT, Revilla C, Munuera L, Sanchez-Sotelo J: Proximal humerus fracture rotational stability after fixation using a locking plate or a fixed-angle locked nail: the role of implant stiffness. Clin Biomech 2010, 25:307-311.

4. Court-Brown CM, Garg A, McQueen MM: The epidemiology of proximal humeral fractures. Acta Orthop 2001, 72:365-371.

5. Fjalestad T, Strømsøe K, Blücher J, Tennøe B: Fractures in the proximal humerus: functional outcome and evaluation of 70 patients treated in hospital. Arch Orthop Trauma Surg 2005, 125:310-316.

6. Vundelinckx BJ, Dierickx CA, Bruckers $L$, Dierickx $C H$ : Functional and radiographic medium-term outcome evaluation of the Humerus Block, a minimally invasive operative technique for proximal humeral fractures. J Shoulder Elbow Surg 2012, 21:1197-1206.

7. Hessmann M, Gotzen L, Gehling H, Baumgaertel F, Klingelhoeffer I: Operative treatment of displaced proximal humeral fractures: two-year results in 99 cases. Acta Chir Belg 1998, 212-219.

8. Jaberg $H$, Warner J, Jakob R: Percutaneous stabilization of unstable fractures of the humerus. J Bone Joint Surg Am 1992, 74:508.

9. Ilchmann $T$, Ochsner $P$, Wingstrand $H$, Jonsson $K$ : Non-operative treatment versus tension-band osteosynthesis in three- and four-part proximal humeral fractures. Int Orthop 1998, 22:316-320.

10. Heers $G$, Torchia M: Shoulder hemi-arthroplasty in proximal humeral fractures. Der Orthopäde 2001, 30:386.

11. Schai P, Imhoff A, Preiss S: Comminuted humeral head fractures: a multicenter analysis. J Shoulder Elbow Surg 1995, 4:319-330.

12. Deeks JJ, Altman DG, Bradburn MJ: Statistical methods for examining heterogeneity and combining results from several studies in metaanalysis. In Systematic Reviews in Health Care: Meta-Analysis in Context. 2nd edition; 2001:285-312.

13. Higgins JP, Thompson SG, Deeks JJ, Altman DG: Measuring inconsistency in meta-analyses. Br Med J 2003, 327:557.

14. DerSimonian R, Laird N: Meta-analysis in clinical trials. Control Clin Trials 1986, 7:177-188. 
15. Mantel N, Haenszel W: Statistical aspects of the analysis of data from retrospective studies of disease. Challenge Epidemiol Issues Selected Readings 2004, 1:533-553.

16. Begg CB, Mazumdar M: Operating characteristics of a rank correlation test for publication bias. Biometrics 1994, 1088-1101.

17. Kristiansen B, Kofoed $\mathrm{H}$ : Transcutaneous reduction and external fixation of displaced fractures of the proximal humerus. A controlled clinical trial. J Bone Joint Surg Br 1988, 70:821.

18. Zyto K, Ahrengart L, Sperber A, Törnkvist H: Treatment of displaced proximal humeral fractures in elderly patients. J Bone Joint Surg Am 1997, 79:412-417.

19. Zyto K, Kronberg M, Broström L-Å: Shoulder function after displaced fractures of the proximal humerus. J Shoulder Elbow Surg 1995, 4:331-336.

20. Fan $Y$, Wang $S$, Luo Y: [Effectiveness comparison of operative and nonoperative treatment for complex proximal humeral fractures in elderly patients]. Zhongguo Xiu Fu Chong Jian Wai Ke Za Zhi 2012, 26:1029.

21. Kollig E, Kutscha-Lissberg F, Roetman B, Dielenschneider D, Muhr G: [Complex fractures of the humeral head: which long-term results can be expected?]. Zentralblatt für Chirurgie 2003, 128:111

22. Zhang MJ, Sun SD, Zhang X, Yang DZ: [The comparisive study of the clinical effect of rotary self-locking intramedullary nail and intramedullary interlocking nail for the treatment of femur fracture]. Zhongguo Gu Shang 2008, 21:766-768.

23. Lepore L, Lepore S, Maffulli N: Intramedullary nailing of the femur with an inflatable self-locking nail: comparison with locked nailing. J Orthop Sci 2003, 8:796-801

24. Knothe U, Knothe Tate ML, Klaue K, Perren SM: Development and testing of a new self-locking intramedullary nail system: testing of handling aspects and mechanical properties. Injury 2000, 31:617-626.

25. Russo R, Cautiero F, Ciccarelli M, Vernaglia Lombardi L: Reconstruction of unstable, complex proximal humeral fractures with the da Vinci cage: surgical technique and outcome at 2 to 6 years. J Shoulder Elbow Surg 2013, 22:422-431.

26. Poeze $M$, Lenssen AF, Van Empel JM, Verbruggen JP: Conservative management of proximal humeral fractures: can poor functional outcome be related to standard transscapular radiographic evaluation? J Shoulder Elbow Surg 2010, 19:273-281.

27. Tauber M, Magosch P, Habermeyer P: [Humeral head replacement in acute proximal humerus fractures]. Der Unfallchirurg 2013

28. Lill H, Katthagen $C$, Jensen $G$, Voigt C: [Arthroscopic fracture management in proximal humeral fractures]. Der Unfallchirurg 2013, 116:296-304.

29. Moreno SG, Sutton AJ, Thompson JR, Ades A, Abrams KR, Cooper NJ: A generalized weighting regression-derived meta-analysis estimator robust to small-study effects and heterogeneity. Stat Med 2012, 31:1407-1417.

doi:10.1186/1749-799X-9-31

Cite this article as: Xu et al.: Avascular necrosis in proximal humeral fractures in patients treated with operative fixation: a meta-analysis. Journal of Orthopaedic Surgery and Research 2014 9:31.

\section{Submit your next manuscript to BioMed Central and take full advantage of:}

- Convenient online submission

- Thorough peer review

- No space constraints or color figure charges

- Immediate publication on acceptance

- Inclusion in PubMed, CAS, Scopus and Google Scholar

- Research which is freely available for redistribution 